\title{
Innovative oxide fuels doped with minor actinides for use in fast reactors
}

\author{
M. Osaka, S. Miwa, K. Tanaka, I. Sato, T. Hirosawa, \\ H. Obayashi, K. Mondo, Y. Akutsu, Y. Ishi, S. Koyama, \\ H. Yoshimochi \& K. Tanaka \\ Fuels and Materials Department, Oarai Research and Development \\ Center, Japan Atomic Energy Agency, Japan
}

\begin{abstract}
Research and development of innovative oxide fuels doped with minor actinides for use in fast reactors are underway. Management of minor actinides in a fast reactor fuel cycle is a key technology for successful realization of a sustainable energy supply with a low impact on the environment. Americium-doped oxide fuels are focused on as representative of minor actinides-containing fuel. Two types of fuels were designed and fabrication tests were carried out. Fundamental properties were also investigated. Results can effectively be reflected to establishment of the fast reactor cycle with minor actinides.

Keywords: fast reactor cycle, minor actinides, oxide fuel, fabrication, characterization, irradiation test, composite fuel.
\end{abstract}

\section{Introduction}

Establishment of a sustainable energy supply for the future is presently being given top consideration. Such energy resources should meet several requirements, including specifically low environmental burden, economical competitiveness, and efficient use of natural resources. In particular, since global warming mainly caused by carbon dioxide $\left(\mathrm{CO}_{2}\right)$ accumulation has become a main concern, energy resources that emit no $\mathrm{CO}_{2}$ or only a small amount of it are strongly expected to be listed at the top among those that satisfy the abovementioned requirements.

Nuclear energy emits a smaller amount of $\mathrm{CO}_{2}$ than the present major energy resources, the fossil energies. Nuclear energy is widely recognized as one of the 
most promising ways to mitigate the global warming problem, although it was not officially recognized by the Kyoto protocol for such a purpose. There are many other advantages from using nuclear energy. It is of special note that the nuclear energy resource becomes available for several hundred years if breeding of new fissile materials, namely plutonium $(\mathrm{Pu})$ and ${ }^{233} \mathrm{U}$, is realized, while the present once-through use of enriched uranium (U) can supply energy only for several tens of years in the coming future. From this viewpoint, a closed nuclear fuel cycle must be established in which the new energy resource $\mathrm{Pu}$ can be effectively utilized.

The fast reactor (FR) is recognized as the most promising candidate for use in such a closed nuclear fuel cycle Sagayama [1]. In this FR cycle, management of minor actinides (MAs), namely neptunium (Np), americium (Am) and curium $(\mathrm{Cm})$, is the key technology for enhancement of reduced environmental burden, effective use of natural resources and non-proliferation of fissile materials. The MAs are generated in spent nuclear fuel. They have high and lasting radiotoxicity, which cause their environmental burden especially for the final disposal of the radioactive wastes. Hence, the MAs should be recovered from the spent nuclear fuel and recycled into reactors. In this way confinement of MAs inside the nuclear fuel cycle is attained. Consequently, radiotoxicity of high level waste is greatly reduced, which can lead to reduced administrative period of final disposal sites for radioactive wastes. The recycling of MAs also contributes to the effective use of natural resources, since MAs yield new high-performance fissile materials other than $\mathrm{Pu}$ nuclides, such as ${ }^{242 \mathrm{~m}} \mathrm{Am}$ and ${ }^{245} \mathrm{Cm}$. The FR has intrinsic advantages in MA-loading into the core because of its flexibility for acceptance of neutron absorbers like MAs, together with higher fission to capture ratio than that in thermal reactors Wakabayashi et al [2]. These advantages should make an effective MA transmutation and utilization possible.

There are, however, tremendous issues to be solved thorough all the nuclear fuel cycle stages for establishment of the FR cycle with MAs. They are mainly caused by the actual difficulties of MA treatment. MA-containing fuels must be fabricated by special apparatuses installed in a radiation-shielded area with remote handling in order to avoid worker exposure, since only several percent of MA addition into fuel raises the radiation dose ten times Casalta et al [3]. The most significant issue for safe reactor operation would be the large impact of MAs on irradiation performances in FRs. Thermal performance values of MAcontaining fuels become degraded due to the lower thermal properties of MA compounds compared with those of the standard fuel components, if a considerable amount of MAs is added to the fuels. Moreover, reprocessing technology of spent MA-containing fuels needs many innovations Sato et al [4]. Thus, MA-management technology is a very challenging topic.

This paper describes some of the challenges faced in establishment of the FR cycle including MAs. Focus is placed on research and development of MAcontaining fuels for use in the FR cycle. A suitable FR cycle scheme is depicted for effective introduction of FRs, together with an optimized MA management strategy. The main fuel used in this FR cycle is an improved version of the existing standard fuel of a mixed oxide $(\mathrm{MOX})$ of $\mathrm{U}$ and $\mathrm{Pu}$, doped with several 
percent of MAs. A second fuel is introduced which is a completely innovative one, namely it is a composite type fuel designed for enhancement of both thermo-physical/chemical and transmutation performances.

\section{Fast reactor-based nuclear fuel cycle system with emphasis on minor actinide management}

Fig. 1 shows a FR-based nuclear fuel cycle system. The system is composed of three sub-cycles, i.e. light water reactor (LWR) sub-cycle, main FR sub-cycle and small FR sub-cycle. FRs are assumed to be gradually introduced and substituted for the present LWRs, starting from around 2050. All Pu, together with all MAs and a part of the fission products (FPs), are recovered from spent nuclear fuel and recycled into FRs. The difference between the two FR subcycles is the forms of MA-containing fuels utilized.

Low-decontaminated fuel (LDF), in which several percent of MAs and FPs are added to the MOX fuel, is used in the main FR sub-cycle Namekawa et al [5]. Since all component elements in the LDF are homogeneously mixed, the MA-containing fuel managed in the main FR sub-cycle is called homogeneous fuel.

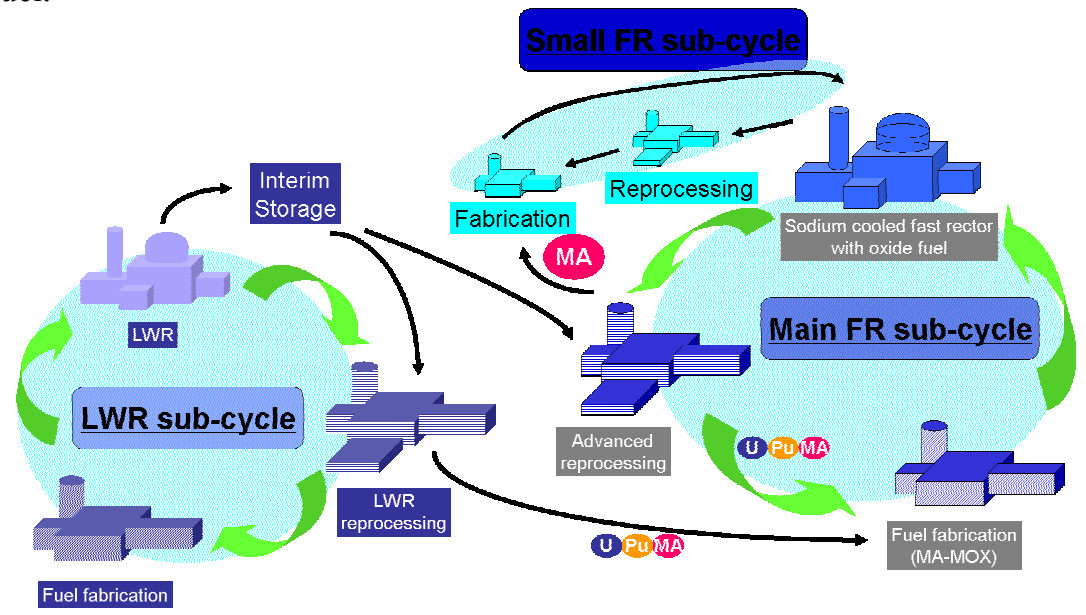

Figure 1: Fast reactor-based fuel cycle system that incorporates minor actinides.

The small FR sub-cycle focuses on rapid incineration of MAs in the introductory period of FRs, since large amounts of MAs have already been accumulated in the present nuclear park. For this purpose, several types of special MA-containing fuel dedicated to the rapid incineration of MAs are designed. Since this special fuel is a composite of MA-compounds and inert matrix (IM), they are called heterogeneous fuel. The heterogeneous fuel is mainly expected to play a supplementary role as a high-performance device for 
rapid incineration of MAs. Therefore, the heterogeneous fuel is incorporated into the main FR sub-cycle, as shown in fig. 1.

Specific elements in the heterogeneous fuel, namely Th and IM elements, as shown later, are concentrated in the small FR sub-cycle. Such concentration can lead to a small impact on the main FR sub-cycle, which helps to get a flexible and smooth attachment/detachment of the small FR sub-cycle to the main FR sub-cycle.

Although many new technologies for the small FR sub-cycle should be developed, e.g. fabrication and reprocessing, they should have adaptability to the existing/standard technologies as long as possible, since the small FR sub-cycle would take place mainly in the introductory phase of the main FR sub-cycle. For this purpose, a fabrication technique based on the traditional powder metallurgy that is typically deployed for the present MOX fabrication is now being developed. Reprocessing is based on the extraction method combined with dissolution of the spent heterogeneous fuel by nitric acid, which are basic technologies adopted for the reprocessing of the present conventional fuel.

The heterogeneous fuels are contained in a special fuel-assembly that contains no LDFs, when they burnup in a FR core with LDFs. Such a fuel-assembly has advantages especially in the reprocessing stage, whereby the heterogeneous fuel can easily be separated from the LDF fuel-assemblies prior to chemical treatments. Although core characteristics should become worse owing to the high absorption cross sections of several IM nuclides, an optimal loading of the heterogeneous fuels was shown to lead to suppression of the degradation of core characteristics to a tolerable level Osaka et al [6].

\section{Innovative oxide fuels for fast reactors}

Americium should be focused on among MAs since Am is important in view of its having a high generation yield in the spent fuel, high and lasting radiotoxicity and expected impacts on various properties of oxide fuels. Therefore, Amcontaining fuel is being studied as a first step to promote research and development of the MA-containing fuels.

\subsection{Homogeneous mixed oxide fuel}

\subsubsection{Fabrication technology with remote operation}

Research and development of Am-containing MOX (Am-MOX), $(\mathrm{U}, \mathrm{Pu}, \mathrm{Am}) \mathrm{O}_{2-\mathrm{x}}$, which is a representative LDF, was started in the early 1990s in a laboratory of Japan Atomic Energy Agency Yoshimochi et al [7]. As almost nothing was known about the effects of Am addition on various characteristics of the standard MOX, we had to grope for the best solution by ourselves. Generally, there are two main tasks for fuel development in the front end of the fuel cycle: establishing fabrication technology and making an optimal design for sound burnup of the fuel.

Fabrication technology for MA-containing fuels is of crucial importance since the fuels must be fabricated in a shielded area by remote operation in order to 
avoid worker exposure. This also means that all related tasks, such as repairing and improving fabrication apparatuses, must be done by remote operation.

Fig. 2 shows laboratory-scale fabrication apparatuses set in a shielded cell in the laboratory. Remote operation by manipulators can make worker exposure negligible.

Since characteristics of Am-MOX fabrication were not known at the beginning, fundamental fabrication tests were carried out Yoshimochi et al [7]. The fabrication procedure was based on the traditional powder metallurgy that is adopted for the standard MOX, as the procedure is relatively simple and compatible with the remote operation. The procedure was composed of powder treatment by rotation milling/mixing of raw powders, compaction by uni-axial pressing, followed by pressureless sintering in a reducing atmosphere. Miwa et al [8] found that Am significantly influenced sintering behaviours of the AmMOX fuel. Thus, optimal sintering atmospheres, together with suitable temperature control patterns, were established for the fabrication of Am-MOX fuel pellets having good qualities such as a high density and a homogeneous structure.

Fabricated pellets were then inserted into a cladding sheath to prepare fuel pins for irradiation tests in a FR. Manufacturing of the fuel pins could be carried out by a semi-automatic remote handling system. The fuel pins were sealed by TIG welding and then inspected using a He-leak detection system for confirmation of the air-tightness/soundness of the fuel pins. Manufactured fuel pins were then assembled into a fuel irradiation rig for the irradiation tests.

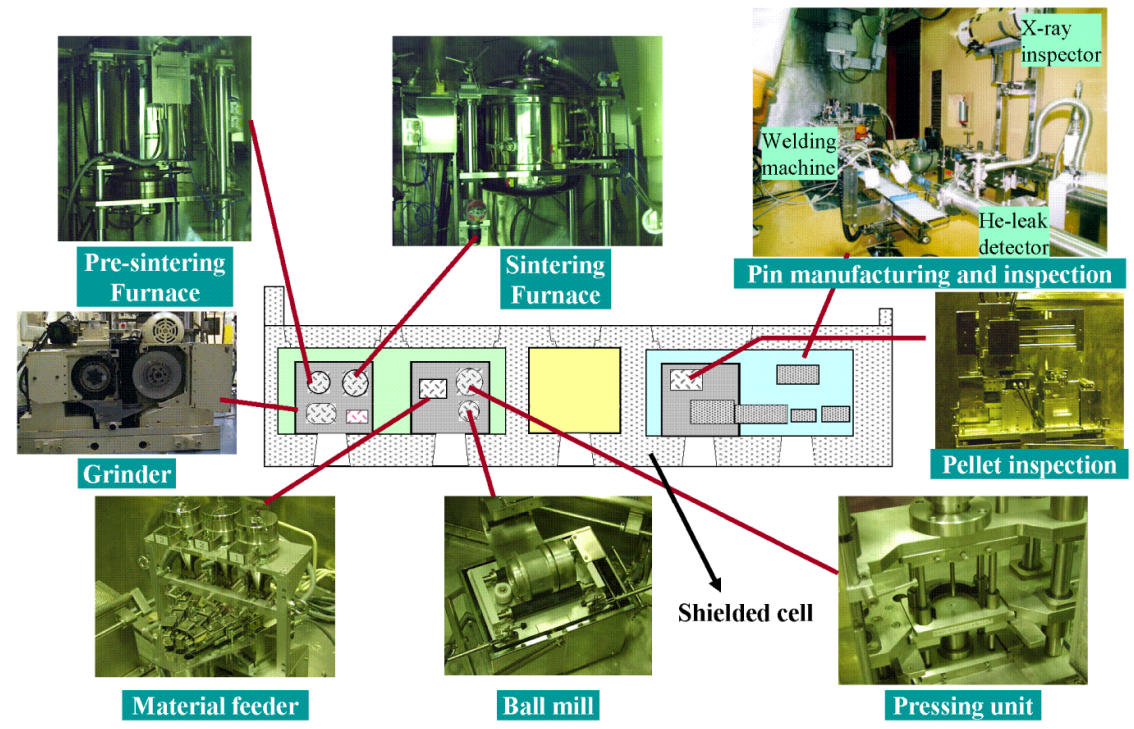

Figure 2: Fabrication system of minor actinide-containing fuel by remote handling. 
One important matter is inspections of the manufactured Am-MOX fuels. Since fuel temperature must be maintained at an appropriate level and cladding integrity must be kept during burnup in order to avoid fuel melting and cladding failure, respectively, impurities in fabricated pellets must be kept at a very low level. This is because a small amount of impurities can degrade thermal properties of the fuel, e.g. lowered melting temperature and thermal conductivity, which can lead to the fuel melting and consequent cladding failure. Therefore, inspection technology for the Am-MOX fuel was established based on that of the standard MOX fuel.

A series of fuel manufacturing test were successfully carried out and a part of the Am-MOX fuels were irradiated in the experimental fast reactor JOYO. This was for the first time in the world that Am-containing fuels were totally manufactured by a remote operation system.

\subsubsection{Optimal design for Am-containing MOX fuel}

The fuel pin design requires much information about fundamental properties of fuels, especially thermophysical properties and irradiation behaviour. Fundamental properties are needed not only for an optimal fuel pin design but also for the above mentioned fuel fabrication technology. Deep understanding of irradiation behaviours via actual irradiation tests in FRs is indispensable because burnup causes various changes/evolutions of fuel properties.

In order to investigate fundamental properties of the Am-MOX fuel, synthesis of Am-MOX from component powder, $\mathrm{UO}_{2}, \mathrm{PuO}_{2}$ and $\mathrm{AmO}_{2}$, was carried out. Since the Am amount was relatively small, $5 \%$ at a maximum, it was assumed that phase behaviour of Am-MOX has similar aspects to that of the standard MOX. Experimental results of X-ray diffraction, thermal analysis and ceramographic observations reported by Osaka et al [9] showed that the AmMOX had a single fluorite phase, (U,Pu,Am) $\mathrm{O}_{2-\mathrm{x}}$, at compositions from near stoichiometry, $x \cong 0$, toward $x$ of about 0.1 . However, several experimental results implied that Am effects on phase behaviour should be well understood if a larger amount of Am is added to the MOX.

Measurement results of melting temperature by Kato et al [10] and thermal conductivity by Morimoto et al [11] showed that Am effects on these properties were not so large, namely both melting temperature and thermal conductivity of Am-MOX with Am content of up to $3.3 \%$ were almost the same level as those of MOX. This was also confirmed analytically by Kurosaki et al [12] in a molecular dynamics simulation. On the other hand, it was revealed that thermodynamic properties were significantly influenced by the addition of Am. Osaka et al [13] found that oxygen potential, which is one of the most important properties that affect almost all bulk properties of oxide fuels, was notably higher than that of the MOX fuel. As oxygen potential dominates the phase behaviour of burnup fuels, thermal properties of a burnup fuel can significantly differ from those of the MOX, if Am content is raised.

To confirm this burnup effect of Am-addition, irradiation tests of Am-MOX fuels in a FR are now being done. Focus is being placed on migration behaviour of Am under a tremendously large temperature gradient in the radial direction of 
the fuel pellet. This behaviour can become a critical issue for the design of Amcontaining MOX fuel, because a possible resultant agglomeration of Am to a specific part of the burnup fuel causes unfavourable property changes, e.g. degraded thermal properties both by direct effect of increased Am content and derived effect via phase changes. Previous irradiation test results showed that such Am migration was similar as that of $\mathrm{Pu}$ Walker and Nicolaou [14]. This implies that no essential improvement is needed for the Am-MOX fuel.

\subsection{Heterogeneous composite fuel}

\subsubsection{Significance}

As mentioned in the previous section, effects of Am-addition on properties of the standard MOX fuel would be significant in the case of larger Am content, although details should be evaluated further. This leads to needs for either improvement of the homogeneous fuel design or introduction of completely innovative fuels. On the other hand, since the requirement of rapid incineration of presently accumulating MAs is of importance as mentioned in section 1, MA content in fuels should be raised. Therefore, novel fuels containing a large amount of MAs were proposed and fundamental studies are underway. Novel fuels are heterogeneous type, namely composites of MA-oxide (host) and IM phases. Such composite fuels have relatively long development history as a highperformance device for incineration of not only MAs but also the considerable amount of $\mathrm{Pu}$ that is generated via dismantling of nuclear weapons, e.g. Degueldre and Yamashita [15]. A characteristic for the present heterogeneous type fuel is its adaptability to the main FR sub-cycle technology as mentioned in section 1. Namely, the main FR sub-cycle is operated with homogeneous fuel and the additional small FR sub-cycle with the heterogeneous fuel is operated for the rapid incineration of MAs, as shown in fig. 1.

Table 1 lists fundamental specifications of heterogeneous fuel. Three types of fuel, namely $\mathrm{MgO}-[16], \mathrm{Si}_{3} \mathrm{~N}_{4}-$ [17] and Mo-based [18], are considered. The host phases are (Pu,Am) $\mathrm{O}_{2-\mathrm{x}}$ for $\mathrm{MgO}$ - and $\mathrm{Si}_{3} \mathrm{~N}_{4}$-based types, while it is (Am,U,Th) $\mathrm{O}_{2-x}$ for Mo-based one. These IMs have been selected so that fuel performance could effectively be enhanced, e.g. higher thermal conductivity, higher chemical/physical stability, and so on.

Table 1: Fundamental specifications of heterogeneous fuel containing minor actinides for the small fast reactor sub-cycle.

\begin{tabular}{|c|c|c|c|c|}
\hline & & MgO-based & $\mathrm{Si}_{3} \mathrm{~N}_{4}$-based & Mo-based \\
\hline \multirow{3}{*}{ Fuel } & Shape & \multicolumn{3}{|c|}{ Sintered columnar pellet } \\
\hline & Density & \multicolumn{3}{|c|}{$>90 \%$ of theoretical } \\
\hline & Form & \multicolumn{3}{|c|}{ Homogeneous composite of host and IM phases } \\
\hline \multirow{3}{*}{$\begin{array}{l}\text { Host } \\
\text { phase }\end{array}$} & Chemical form & \multicolumn{2}{|c|}{$(\mathrm{Pu}, \mathrm{Am}) \mathrm{O}_{2-x}$} & $(\mathrm{Am}, \mathrm{U}, \mathrm{Th}) \mathrm{O}_{2-x}$ \\
\hline & Weight fraction & $0.3-0.5$ & $0.16-0.20$ & $0.5-0.9$ \\
\hline & $\begin{array}{l}\text { Morphologic } \\
\text { shape }\end{array}$ & Sphere, $\sim \varphi 100 \mu \mathrm{m}$ & $\begin{array}{c}\text { Grain boundary } \\
\text { phase }\end{array}$ & Particle, $<\varphi 10 \mu \mathrm{m}$ \\
\hline $\begin{array}{l}\text { IM } \\
\text { phase }\end{array}$ & Chemical form & $\mathrm{MgO}$ & $\mathrm{Si}_{3} \mathrm{~N}_{4}$ & $\begin{array}{c}\text { Mo } \\
\text { (recovered from } \\
\text { spent nuclear fuel) }\end{array}$ \\
\hline
\end{tabular}


An important point for the selection of IM compound was adaptability to the main FR sub-cycle technology, especially the fabrication process. The fabrication process should be simple so that the remote operation can be applied, the same as for the case of Am-MOX fabrication. Fundamental studies for the fabrication and characterization of the heterogeneous fuels are described next.

\subsubsection{Fabrication process and fundamental properties}

Basic studies for establishment of fabrication process were carried out by using surrogate material, $\mathrm{CeO}_{2}$, which has similar properties to those of $\mathrm{PuO}_{2}$ or $\mathrm{AmO}_{2}$. Fig. 3 shows fundamental fabrication process for heterogeneous fuels Osaka et al [19]. The process was basically that of the traditional powder metallurgy for the standard MOX fuel. This means that the standard apparatuses can immediately be installed into the fabrication facility for the heterogeneous fuel without any essential improvements. Appearances of fabricated pellets of $\mathrm{CeO}_{2}-\mathrm{MgO}$ and $\mathrm{CeO}_{2}-\mathrm{Mo}$ by the present process are also shown in fig. 3 . There were neither cracks nor visible defaults in the sintered bodies. Densities attained for the sintered pellets were more than $90 \%$ of theoretical.

Characterizations of host phases are another important topic. Oxygen potential of the host phase of Mo-based fuel, (Am,U,Th) $\mathrm{O}_{2-\mathrm{x}}$, were predicted by Osaka et al [20] using the chemical thermodynamic method. A small amount of $\mathrm{U}$ addition was revealed to be effective for optimization of oxygen potentials during burnup. Defect structures of a $\mathrm{ThO}_{2}$-based solid solution were also investigated with the aid of a molecular dynamics simulation.

Regarding the host phase of $\mathrm{MgO}-$ and $\mathrm{Si}_{3} \mathrm{~N}_{4}$-based fuels, experimental investigations on a $(\mathrm{Pu}, \mathrm{Am}) \mathrm{O}_{2-\mathrm{x}}$ solid solution were carried out. Experimental investigations on the phase relation by Miwa et al [21] and oxygen potential by Osaka et al [22] have shown that oxygen non-stoichiometry should be optimized for this system.
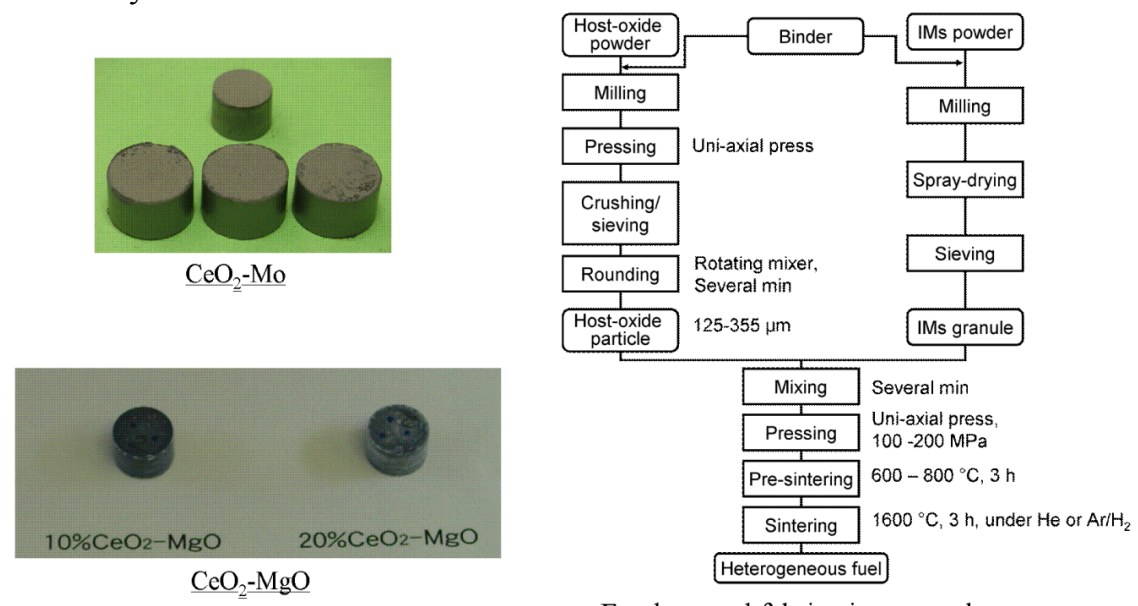

Fundamental fabrication procedure

Figure 3: Fundamental fabrication process for heterogeneous fuel and appearances of surrogate $\mathrm{CeO}_{2}-\mathrm{MgO}$ and $\mathrm{CeO}_{2}-\mathrm{Mo}$ fuels. 


\section{Concluding remarks}

Innovative oxide fuels doped with minor actinides for use in fast reactors are being developed. Two types of fuels, namely homogeneous and heterogeneous ones for the main and small fast reactor sub-cycles were proposed. Research and development of Am-doped fuels is underway as a first step. Novel and valuable results have been obtained, which can effectively be reflected to the further promotion of the minor actinide management technology in the fast rector cycle system.

\section{References}

[1] Sagayama, Y., Feasibility study on commercialized fast reactor cycle systems (1) current status of the phase-II study. Proc. of GLOBAL2005, Tsukuba, paper No. 380, 2005.

[2] Wakabayashi, T., Takahashi, K. \& Yanagisawa, T., Feasibility studies on plutonium and minor actinide burning in fast reactors. Nuclear Technology, 118, pp. 14-18, 1997.

[3] Casalta, S., Matzke, Hj. \& Prunier, C., A thermodynamic properties study of the americium-oxygen system. Proc. of Int. Conf. GLOBAL95, Versaille, pp. 1-9, 1995.

[4] Sato, K., Koma, Y., Nakabayashi, H., Yano, K., Amamoto, I., Kitajima, S., Kobayashi, T., Higuchi, T., Nakanishi, S. \& Yoshiuji, T., Conceptual design study and evaluation of advanced reprocessing plants in the feasibility study on commercialized FR cycle systems in Japan. Proc. of GLOBAL2005, Tsukuba, paper No. 502, 2005.

[5] Namekawa, T., Kawaguchi, K., Koike, K., Haraguchi, S. \& Ishii, S., Conceptual design study and evaluation of advanced fuel fabrication systems in the feasibility study on commercialized FR fuel cycle in Japan. Proc. of GLOBAL2005, Tsukuba, paper No. 424, 2005.

[6] Osaka, M., Takano, S., Yamane, Y. \& Misawa, T., On the fast reactor cycle scheme that incorporates a thoria-based minor actinide-containing cermet fuel. Submitted to Progress in Nuclear Energy.

[7] Yoshimochi, H., Nemoto, M., Koyama, S. \& Namekawa, T., Fabrication technology for MOX fuel containing $\mathrm{AmO}_{2}$ by an in-cell remote process. Journal of Nuclear Science and Technology, 41(8), pp. 850-856, 2004.

[8] Miwa, S., Osaka, M., Yoshimochi, H., Tanaka, K., Seki, T. \& Sekine, S., Effect of oxygen potential on the sintering behavior of MOX fuel containing Am. Recent Advances in Actinide Science, eds. Alvarez, R., Bryan, N.D. \& May, I., RSC Publishing: Manchester, pp. 400-402, 2006.

[9] Osaka, M., Miwa, S., Yoshimochi, H., Tanaka, K., Kurosaki, K. \& Yamanaka, S., An experimental investigation of effects of americium addition to $(\mathrm{U}, \mathrm{Pu}) \mathrm{O}_{2-\mathrm{x}}$ on phase relation. Recent Advances in Actinide Science, eds. Alvarez, R., Bryan, N.D. \& May, I., RSC Publishing: Manchester, pp. 406-408, 2006. 
[10] Kato, M., Morimoto, K., Kashimura, M. \& Abe, T., Measurements of melting points in the $\mathrm{PuO}_{2}-\mathrm{UO}_{2}$ system. Int. Information Exchange Mtg. Thermodyn. Nucl. Fuels, Saclay, 2006.

[11] Morimoto, K., Kato, M., Kashimura, M. \& Abe T., Thermal conductivity of (U,Pu, Am) $\mathrm{O}_{2-\mathrm{x}}$ solid solutions. Int. Information Exchange Mtg. Thermodyn. Nucl. Fuels, Saclay, 2006.

[12] Kurosaki, K., Adachi, J., Katayama, M., Osaka, M., Tanaka, K., Uno, M. \& Yamanaka, S., Molecular dynamics studies of americium-containing mixed oxide fuels. Journal of Nuclear Science and Technology, 43(10), pp. 1224-1227, 2006.

[13] Osaka, M., Sato, I., Namekawa, T., Kurosaki, K. \& Yamanaka, S., Oxygen potentials of $\left(\mathrm{U}_{0.685} \mathrm{Pu}_{0.270} \mathrm{Am}_{0.045}\right) \mathrm{O}_{2-\mathrm{x}}$ solid solutions. Journal of Alloys and Compounds, 397, pp. 110-114, 2005.

[14] Walker, C.T. \& Nicolaou, G., Transmutation of neptunium and americium in a fast neutron flux: EPMA results and KORIGEN predictions for the superfact fuels. Journal of Nuclear Materials, 218, pp. 129-138, 1995.

[15] Degueldre, C. \& Yamashita, T., Inert matrix fuel strategies in the nuclear fuel cycle: the status of the initiative efforts at the 8 th inert matrix fuel workshop. Journal of Nuclear Materials, 319, pp. 1-5, 2003.

[16] Osaka, M., Serizawa, H., Kato, M., Nakajima, K., Tachi, Y., Kitamura, R., Miwa, S., Iwai, T., Tanaka, K., Inoue, M. \& Arai, Y., Research and development of minor actinide-containing fuel and target in a future integrated closed cycle system. Journal of Nuclear Science and Technology, in press.

[17] Yamane, J., Imai, M. \& Yano, T., Fabrication and basic characterization of silicon nitride as an inert matrix. submitted to Progress in Nuclear Energy.

[18] Osaka, M., Koi, M., Takano, S., Misawa, T. \& Yamane, Y. A novel concept for americium-containing target for use in fast reactors, Journal of Nuclear Science and Technology, 43(4), pp. 367-374, 2006.

[19] Osaka, M., Miwa, S. \& Tachi, Y., Simple fabrication process for $\mathrm{CeO}_{2}$ $\mathrm{MgO}$ composite as surrogate for actinide-containing target for use in nuclear fuel. Ceramic International, 32, pp. 659-663, 2006.

[20] Osaka, M., Kurosaki, K. \& Yamanaka, S., Prediction of oxygen potential in americium thorium oxides phase of a cermet fuel. Journal of Nuclear Materials, in press.

[21] Miwa, S., Osaka, M., Yoshimochi, H., Tanaka, K., Kurosaki, K., Uno, M. \& Yamanaka, S., Phase behavior of $\mathrm{PuO}_{2-\mathrm{x}}$ with addition of $9 \% \mathrm{Am}$. Journal of Alloys and Compounds, in press.

[22] Osaka, M., Kurosaki, K. \& Yamanaka, S., Oxygen potential of $\left(\mathrm{Pu}_{0.91} \mathrm{Am}_{0.09}\right) \mathrm{O}_{2-\mathrm{x}}$. Journal of Nuclear Materials, 357, pp. 69-76, 2006. 